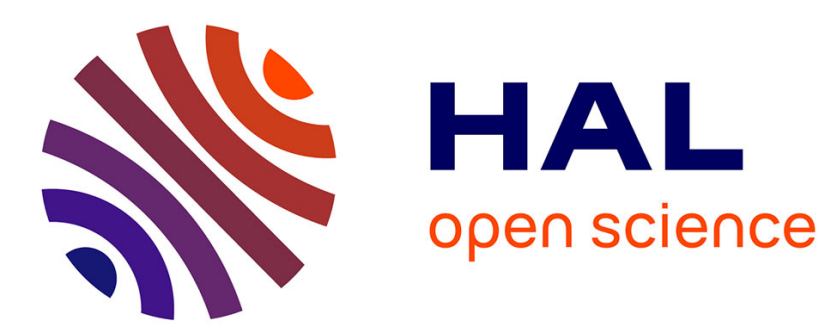

\title{
Mexique: le sombrero de Mexico se réduit relativement
}

Laurent Chalard, Gérard-François Dumont

\section{To cite this version:}

Laurent Chalard, Gérard-François Dumont. Mexique : le sombrero de Mexico se réduit relativement. Population et avenir, 2008, 688, pp.4-7 et 20. 10.3917/popav.688.0004 . halshs-01250675

\section{HAL Id: halshs-01250675 \\ https://shs.hal.science/halshs-01250675}

Submitted on 5 Jan 2016

HAL is a multi-disciplinary open access archive for the deposit and dissemination of scientific research documents, whether they are published or not. The documents may come from teaching and research institutions in France or abroad, or from public or private research centers.
L'archive ouverte pluridisciplinaire HAL, est destinée au dépôt et à la diffusion de documents scientifiques de niveau recherche, publiés ou non, émanant des établissements d'enseignement et de recherche français ou étrangers, des laboratoires publics ou privés. 


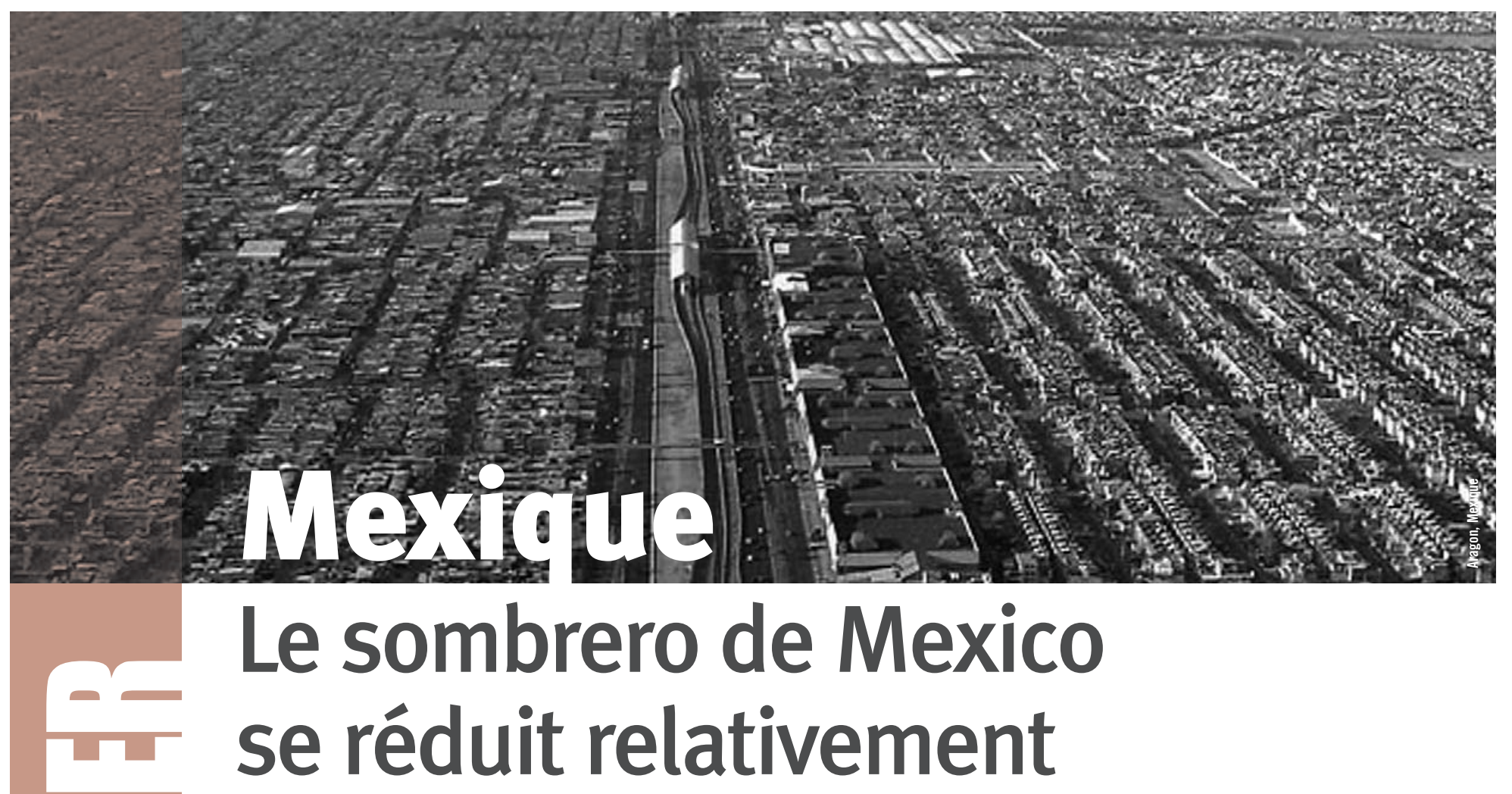

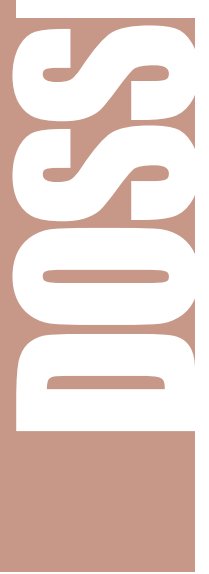

Pendant longtemps, nombre de projections annonçaient 30 millions d'habitants pour l'agglomération de Mexico dès l'an 2000. Le quart des Mexicains allait se concentrer dans la ville capitale, dont l'agglomération devait écraser l'armature urbaine ${ }^{1} \mathrm{du}$ Mexique $^{2}$. Or, il n'en est rien. Que s'est-il passé ? Les résultats du dernier recensement, qui indiquent pour le Mexique une population de 103,2 millions d'habitants, permettent de répondre à cette question.

par Laurent CHALARD et Gérard-François DUMONT*

* Université de Paris-Sorbonne.

1. "L'armature urbaine " est la façon dont se structurent les villes au sein d'un terrioire considéré.

2. Rappelons que le Mexique s'intitule officiellement États-Unis du Mexique, de 31 États exactement plus un district fédéral, et que sa constitution est fédérale.

3. Dans le contexte général dune nette decelération de que du Mexique, du fait de la que du Mexique, du fait de la tion massive et de la baisse de la fécondité. Cf. Wackermann, Gabriel (Direction), L'Amérique latine, Paris, Ellipses, 2005 et Wackermann, Gabriel (Direction), tations corrigées, Paris, Ellipses, 2005

4. Comme ailleurs dans le monde, la délimitation constitue un probleme majeur pour pouvoir determiner la hierarchie des agglomérations et cón arr leur dyanique internet wwwineri do mx), institut statisticue du Mexique, a cré en 2004 le concept de « zones

n effet, depuis les années 1990, se constate au Mexique un ralentissement de la croissance démographique de la capitale ${ }^{3}$ et le renforcement relatif des autres agglomérations.

\section{L'ORGANISATION DU TERRITOIRE} AU MEXIOUE

\section{Dénomination \\ mexicaine}

Entidades

Municipios

Zonas metropolitanas Zones métropolitaines

Localités

187904

Manzanas urbanas

Nombre d'entités

32

2454

55

Localidades

Quartiers urbains

1229745

\section{La géographie des agglomérations de plus de 200000 habitants}

Le Mexique compte un fort taux d'urbanisation, avec une proportion de $75 \%$ de population urbaine dans l'ensemble de la population, un pourcentage équivalent à celui de la France $(75,5 \%)$. L'émigration rurale ne représente plus que des flux assez faibles, sauf dans le Sud, et se dirige souvent vers les États-Unis plutôt que vers des villes mexicaines.

L'armature urbaine compte une soixantaine d'agglomérations 4 de plus de 200000 habitants, où vit 55,1\% de la population du Mexique selon le dernier recensement (2005) contre $51,2 \%$ en 1990 , soit une progression de près de quatre points en quinze ans. Aucune des agglomérations qui comptaient déjà plus de 200000 habitants en 1990 n'a vu sa population diminuer entre 1990 et 2005 , et une seule entre 2000 et 2005 .

2. L'ÉVOLUTION DE LA POPULATION DES AGGLOMÉRATIONS DE PLUS DE 200000 HABITANTS AU MEXIOUE

\begin{tabular}{|c|c|c|c|c|}
\hline & 1990 & 1995 & 2000 & 2005 \\
\hline $\begin{array}{l}\text { Population des } \\
\text { agglomérations } \\
\text { de plus de } 200000 \\
\text { habitants }\end{array}$ & 41584821 & 48106703 & 52198797 & 56885008 \\
\hline Part dans le pays & $51,2 \%$ & $52,8 \%$ & $53,6 \%$ & $55,1 \%$ \\
\hline $\begin{array}{l}\text { Population totale } \\
\text { du Mexique (nom- } \\
\text { bre d'habitants) }\end{array}$ & 81249645 & 91158290 & 97483412 & 103263288 \\
\hline
\end{tabular}

La géographie de l'armature urbaine apparaît déséquilibrée, car les agglomérations sont inégalement réparties sur le territoire national. La majorité des grandes villes se situe dans le Nord et le Centre du pays, alors que le Sud en apparaît relativement dépourvu, du fait de la différence dans les taux d'urbanisation, élevés au Nord, plus faibles au Sud.

- Le centre du Mexique a la plus forte concentration d'agglomérations sur les hautes terres centrales, les plus

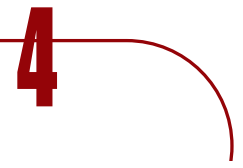


densément peuplées, avec une grande grappe de métropoles le long d'un axe allant de l'agglomération de Veracruz à celles de Guadalajara et d'Aguascalientes, en passant par Puebla, Mexico, et Leon, soit une douzaine d'agglomérations comptant plus de 200000 habitants.

Le Sud, moins urbanisé, compte en général une seule agglomération de plus de 200000 habitants par État (Cancun, Merida, Villahermosa, Tuxtla Guttierez, Oaxaca), sauf dans celui de Veracruz où les fonctions portuaires et l'exploitation pétrolière ont fait émergé une grappe de villes.

Au Nord, les agglomérations comptant plus de 200000 habitants sont centrées sur la côte Pacifique (avec cinq agglomérations dans les États maritimes de Sonora et de Sinaloa, dont les villes de Hermosillo et Culiacán, en retrait du littoral, au milieu des plaines côtières agricoles) ou à proximité de la frontière américaine au nord-ouest (trois agglomérations dans l'État de Basse-Californie du Nord, dont Tijuana) et au nord-est (six agglomérations autour de Monterrey).

\section{UNE COMPARAISON DE LA RÉPARTITION DE LA POPULATION DU MEXIQUE}

ET DE LA FRANCE SELON L'ARMATURE URBAINE

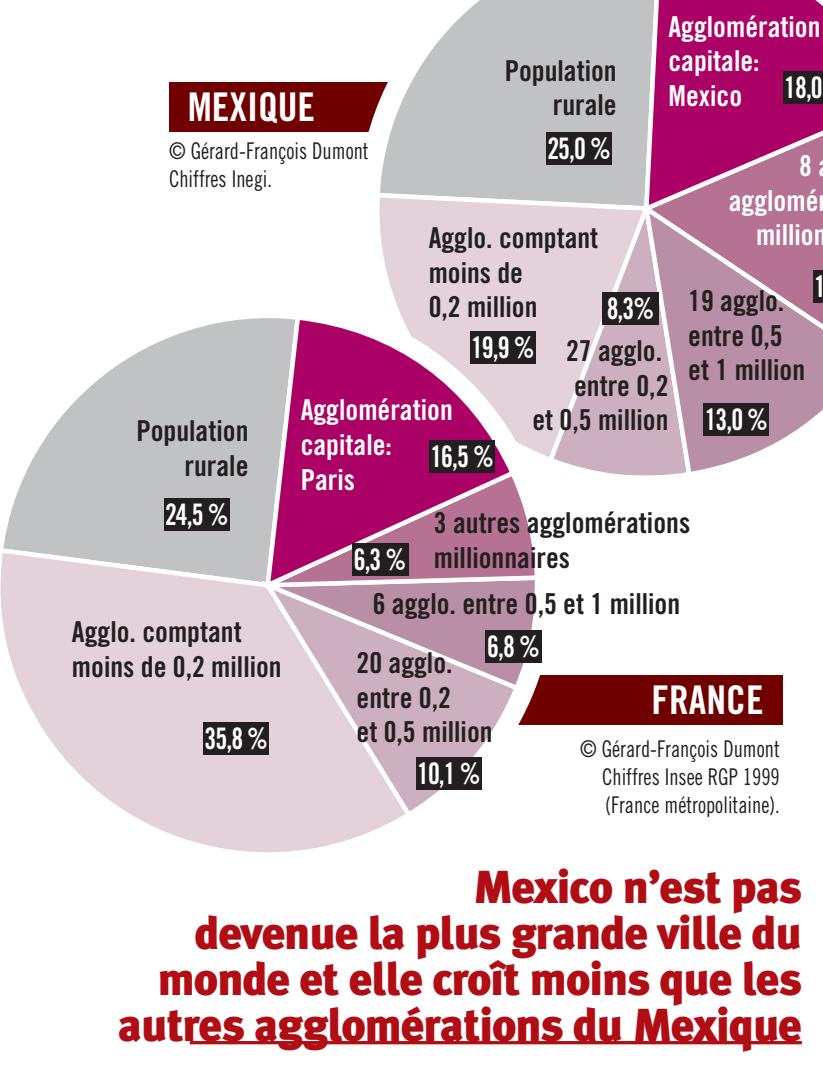

La zone métropolitaine de Mexico, après le ralentissement très sensible de sa croissance, compte seulement 19,2 millions d'habitants selon la définition de l'INEGI'. Mexico n'est pas devenue, comme cela a souvent été annoncé, l'agglomération la plus peuplée du monde. Elle n'a pas atteint les 30 millions d'habitants en l'an 2000, comme souvent escompté dans les années 1980 avec des projections basées de façon erronée sur une croissance linéaire, mais elle se classe troisième après Tokyo et New York. Elle demeure la première agglomération d’Amérique latine, légèrement devant São Paulo.
4. LA POPULATION DE LA ZONE MÉTROPO-

LITAINE DE MEXICO ET SA PROPORTION DANS LA POPULATION TOTALE DU MEXIQUE

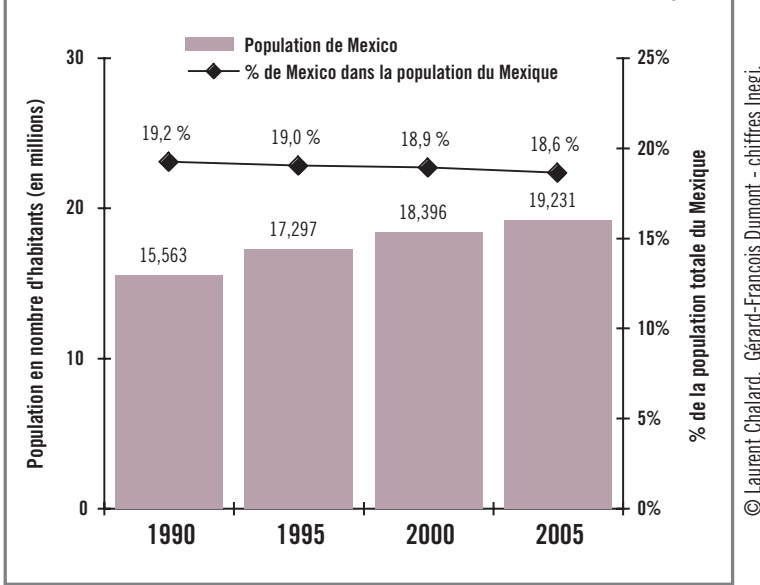

L'importance démographique de Mexico est due à ses fonctions de capitale politique, culturelle, économique et industrielle du Mexique, sachant que Mexico a toujours été une ville importante dès l'époque Aztèque, sous le nom de Tenochtitlan. Une tendance de l'armature urbaine du Mexique à la macrocéphalie ${ }^{7}$ apparaît donc puisque, en 2005, Mexico est 4,5 fois plus peuplée que la deuxième agglomération, Guadalajara, mais cette tendance est nettement moindre que dans nombre de pays d'Amérique latine, comme en Argentine avec Buenos Aires ou au Chili avec Santiago, deux capitales qui concentrent le tiers de la population de leur pays. En outre, cette tendance s'estompe.

\section{Mexico désormais concurrencée par... les États-Unis}

En effet, Mexico compte une croissance moindre que le pays $(23,6 \%$ entre 1990 et 2005 contre $27,1 \%$ entre 1990 et 2005), mais encore importante en volume, près de 4,5 millions d'habitants en quinze ans. Son pourcentage dans la population du pays se réduit à chaque recensement depuis 1990, passant de 19,2\% en 1990 à 18,6\% en 2005 . Cette faible croissance relative de la capitale est liée à deux facteurs. D'une part, sa croissance naturelle se ralentit avec une fécondité plus faible que la moyenne mexicaine, même si son taux d'accroissement naturel est assez élevé, en raison d'une structure par âge favorable. D'autre part, de 2000 à 2005 , le solde migratoire de Mexico est négatif, avec une spécificité, un solde migratoire négatif avec l'étranger. Depuis les années 1990, Mexico est non seulement réceptrice de flux migratoires moins importants en provenance du reste du Mexique, et désormais essentiellement du centre-sud, mais elle est devenue une terre d'émigration. Autrement dit, Mexico n'est plus la « pompe aspirante du pays ", car les régions traditionnelles d'émigration vers la capitale, qu'étaient les hautes terres centrales comme le Michoacán, sont aujourd'hui des régions d'émigration vers les États-Unis. En outre, devant le développement économique insuffisant de Mexico, des populations préfèrent partir aux États-Unis, phénomène nouveau, car auparavant, l'émigration vers les États-Unis était essentiellement rurale $^{8}$. métropolitaines ». Sa définition des délimitations des agglomérations prête cependant à discussion car elle varie pour chaque "zone métropolitaine " définie. Certaines se composent seulement de communes en continuité de bâti, d'autres de communes proches intégrées fonctionnellement à l'agglomération, d'autres de communes au taux de croissance important ou incluses dans un programme de planification sans être rattachées fonctionnellement à l'agglomération. Pour définir les agglomérations, nous avons choisi de ne reteni que les communes en continuité du bâti, délimitation proche de celle des unités urbaines françaises. En outre, pour trois agglomérations du nord (dont Mexicali), où la municipalité est beaucoup plus étendue que lasgome retenu que la population de la

5. La «zone métropolitaine » de Mexico comprend tous les types de communes susmentionnés. La population de l'agglomération, de 18,6 millions de personnes en 2005 selon notre délimitation, est légèrement moins importante.

6. Dumont, Gérard-François, Les populations du monde, 


\section{Une croissance supérieure à celle de la capitale pour les huit agglomérations millionnaires}

Le Mexique possède une armature de grandes villes ${ }^{9}$ avec, outre la capitale, huit agglomérations millionnaires en population (contre, par comparaison, trois en France). Sur les dernières périodes intercensitaires, la croissance démographique moyenne de ces huit agglomérations millionnaires est double de celle de Mexico. Parmi elles, cinq n'étaient d'ailleurs pas millionnaires en habitants en 1990. Les huit agglomérations millionnaires se concentrent au centre, dont les plus peuplées, et au nord. En revanche, aucune ne se localise au sud du pays à l'urbanisation plus faible.

\section{LA CROISSANCE DÉMOGRAPHIQUE} DES AGgLOMÉRATIONS DU MEXIQUE SELON LES STRATES
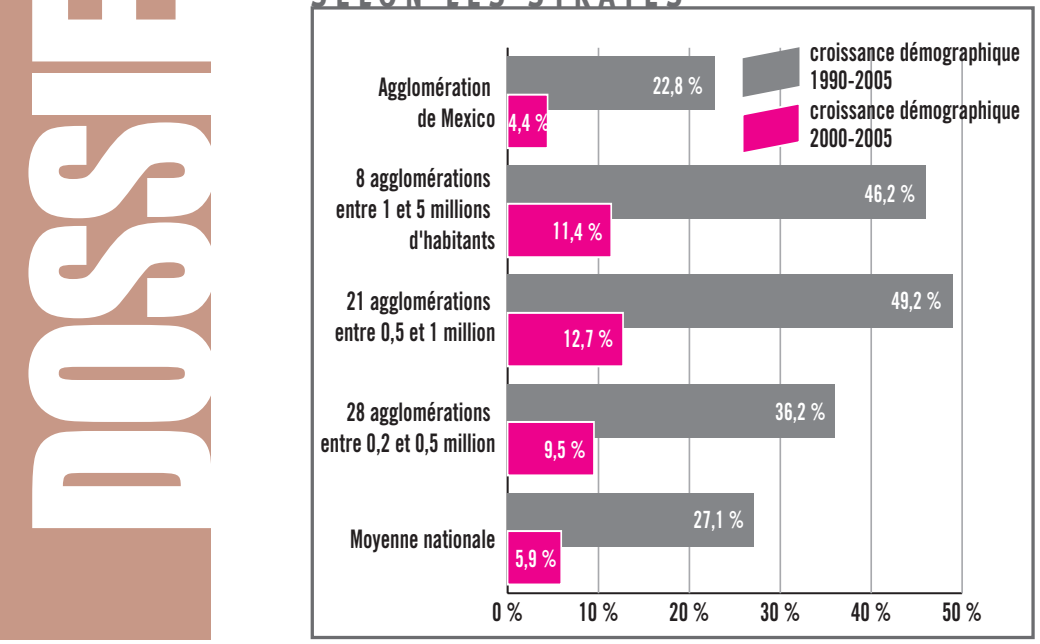

6. LES 28 AGGLOMÉRATIONS LES PLUS PEUPLÉES DU MEXIQUe

\begin{tabular}{|c|c|c|c|c|c|c|}
\hline & 욘 & $\begin{array}{l}\text { Agglomérations comptant } \\
\text { plus de } 500000 \text { habitants }\end{array}$ & État & $\begin{array}{c}\text { Population } \\
1990\end{array}$ & $\begin{array}{l}\text { Population } \\
2005\end{array}$ & $\begin{array}{l}\text { Croissance } \\
2005 / 1990\end{array}$ \\
\hline 1 mégapole & 1 & Vallée de Mexico & & 15162488 & 18620763 & $22,8 \%$ \\
\hline 3 aggomérations & 2 & Guadalajara & JALISCO & 2977126 & 4060531 & $36,4 \%$ \\
\hline \multirow[t]{2}{*}{ plurimillionnaires } & 3 & Monterrey & NUEVO LEON & 2613227 & 3664331 & $40,2 \%$ \\
\hline & 4 & Puebla-Tlaxcala & PUEBLA & 1458099 & 2109049 & $44,6 \%$ \\
\hline \multirow{5}{*}{$\begin{array}{l}5 \text { agglomérations } \\
\text { millionnaires }\end{array}$} & 5 & Tijuana & BASSE-CALIFORNIE NORD & 747381 & 484005 & $98,6 \%$ \\
\hline & 6 & Toluca & MEXICO & 928968 & 426935 & $53,6 \%$ \\
\hline & 7 & Juárez & CHIHUAHUA & 798499 & 313338 & $64,5 \%$ \\
\hline & 8 & León & GUANAUUATO & 867920 & 278087 & $47,3 \%$ \\
\hline & 9 & Torreon-La Laguna & COAHUILA & 791891 & 1011183 & $27,7 \%$ \\
\hline \multirow{22}{*}{$\begin{array}{l}19 \text { aggloméra- } \\
\text { tions comptant } \\
\text { plus de } 500000 \\
\text { habitants et } \\
\text { moins de } 1 \text { mil- } \\
\text { lion au recense- } \\
\text { ment de } 2005\end{array}$} & 10 & San Luis Potosí & SAN LUIS POTOSI & 658712 & 957753 & $45,4 \%$ \\
\hline & 11 & Queretaro & QUERETARO & 555491 & 918100 & $65,3 \%$ \\
\hline & 12 & Mérida & YUCATAN & 620646 & 886188 & $42,8 \%$ \\
\hline & 13 & Tampico & TAMAULIPAS-VERACRUZ & 648598 & 803196 & $23,8 \%$ \\
\hline & 14 & Cuernavaca & MORELOS & 539425 & 787556 & $46,0 \%$ \\
\hline & 15 & Acapulco & GUERRERO & 653973 & 786830 & $20,3 \%$ \\
\hline & 16 & Chihuahua & CHIHUAHUA & 530783 & 758791 & $43,0 \%$ \\
\hline & 17 & Aguascalientes & AGUASCALIENTES & 506274 & 723043 & $42,8 \%$ \\
\hline & 18 & Saltillo & COAHUILA & 469166 & 705637 & $50,4 \%$ \\
\hline & 19 & Morelia & MICHOACAN & 492901 & 684145 & $38,8 \%$ \\
\hline & 20 & Veracruz & VERACRUZ & 473156 & 654216 & $38,3 \%$ \\
\hline & 21 & Mexicali & BASSE-CALIFORNIE NORD & 438377 & 653046 & $49,0 \%$ \\
\hline & 22 & Villahermosa & TABASCO & 437567 & 644629 & $47,3 \%$ \\
\hline & 23 & Hermosillo & SONORA & 406417 & 641791 & $56,3 \%$ \\
\hline & 24 & Culiacan & SINALOA & 415046 & 605304 & $45,8 \%$ \\
\hline & 25 & Tuxtla-Gutiérrez & CHIAPAS & 340751 & 576872 & $69,3 \%$ \\
\hline & 26 & Cancún & QUINTANA ROO & 176765 & 572973 & $212,8 \%$ \\
\hline & 27 & Reynosa & TAMAULIPAS & 282667 & 526888 & $68,2 \%$ \\
\hline & 28 & 0axaca & OAXACA & 331247 & 504159 & $52,2 \%$ \\
\hline & & TOTAL des 28 agglomérations & & 35323561 & 48359339 & $36,9 \%$ \\
\hline & & POPULATION du Mexique & & \begin{tabular}{|l}
81249645 \\
\end{tabular} & 103263288 & $27,1 \%$ \\
\hline & & $\%$ des 28 agglomérations & & $43,5 \%$ & $46,8 \%$ & \\
\hline
\end{tabular}

\section{Les deux agglomérations comptant environ 4 millions d'habitants}

Les deux agglomérations les plus peuplées du Mexique après Mexico, Guadalajara et Monterrey, avoisinent les quatre millions d'habitants, soit la dimension de Milan ou de Barcelone en Europe. Elles ont émergé à la fin du XIXe siècle et sont les seules à avoir une influence suprarégionale en dehors de Mexico. Néanmoins, leur spécificité économique est différente. Guadalajara, métropole du centre-ouest du Mexique, se présente plutôt comme un grand centre de services et de commerces. À l'origine, elle n'est pas une grande ville industrielle, même si elle dispose d'un tissu de PME, notamment dans la chaussure et l'agroalimentaire, et connaît un développement récent dans la haute technologie, en particulier dans l'informatique (IBM, Hewlett Packard, Intel...). Monterrey, au nord-est, est la deuxième métropole industrielle du pays après Mexico et bénéficie de sa relative proximité avec les États-Unis (140 km). Elle est considérée comme ayant le plus haut PNB par habitant d'Amérique centrale et la ville la plus sure. Sa prospérité se base sur l'importance du secteur privé dans l'industrie lourde (sidérurgie, cimenteries, métallurgie, verrerie) et dans des activités diversifiées (agroalimentaire, commerces et services); son agglomération ne possède guère de maquiladoras ${ }^{10}$.

\section{Deux autres agglomérations millionnaires proches de Mexico}

Derrière ces deux métropoles relativement indépendantes de Mexico, Puebla et Toluca croissent en revanche dans la logique de la capitale, bénéficiant des décentralisations d'activités industrielles, car elles offrent de l'espace face à la saturation relative de l'espace dans la vallée de Mexico. Puebla, à deux heures de Mexico par l'autoroute, a des activités dans la mécanique (usine Volkswagen, la plus grande du pays), le textile, l'agroalimentaire et un peu d'informatique. Toluca, à l'ouest de Mexico, doit notamment son développement à ses industries mécaniques (dont l'automobile : usine BMW) et à l'agroalimentaire, et dispose d'une vaste zone industrielle le long de l'autoroute allant vers Mexico. Son aéroport possède aussi un terminal international.

\section{Deux agglomérations millionnaires à la frontière états-unienne}

Parmi les nouvelles agglomérations millionnaires depuis 1990, deux se trouvent à la frontière états-unienne : Tijuana et Ciudad Juarez. Tijuana, ville-jumelle de San Diego, a vu sa population doubler en quinze ans. Sa croissance, la plus forte des agglomérations de plus de 200000 habitants, après Cancun, est le produit du développement des maquiladoras, mais aussi d'activités frontalières particulières (prostitution, activités illicites...). En outre, elle constitue une étape avant de passer la frontière étatsunienne. Tijuana, nouvelle agglomération millionnaire depuis 1995, en a dépassé deux autres, Leon, la capitale de la chaussure, sur la route des États-Unis, et Torreon. La croissance de Ciudad Juarez a la même origine que celle de Tijuana, la ville-jumelle états-unienne étant El Paso. 


\section{La plus forte croissance dans les métropoles intermédiaires}

Dans l'armature urbaine des métropoles, le renforcement de la hiérarchie intermédiaire ${ }^{11}$ est net avec, depuis 2005 , 19 agglomérations comprenant entre 500000 et un million d'habitants, dont onze ne faisaient pas partie de cette catégorie en 1990. Leur croissance moyenne, pour l'ensemble de la période 1990-2005, est légèrement supérieure à celle des agglomérations de plus de 1 million d'habitants, et donc très largement supérieure à la moyenne nationale. La répartition géographique de ces 19 métropoles intermédiaires dans le territoire du Mexique est plus égalitaire, la plupart étant capitales d'État et exerçant leur influence à l'échelle de cet État. Sept sont localisées dans le Sud du Mexique, six dans le Centre et six autres dans le Nord. Certaines sont des villes anciennes, d'autres plus récentes. Sept de ces 19 agglomérations ont enregistré entre 1990 et 2005 une croissance particulièrement importante, supérieure à $50 \%$ : trois au Nord, une au Centre, et trois au Sud.

La croissance la plus forte s'est constatée pour Cancun, à l'extrême-est, près de 600000 habitants en 2005 , dont la population a plus que triplé en l'espace de quinze ans, grâce au développement du tourisme balnéaire, qui en fait une des destinations les plus prisées des Caraïbes.

Six parmi ces 19 métropoles intermédiaires comptent une croissance démographique sur quinze ans entre 50 et $70 \%$, à commencer par Reynosa, 634000 habitants, ville frontière au nord-est, qui a presque doublé sa population, et dont l'économie combine maquiladoras et pétrochimie. Trois autres, Queretaro, Hermosillo et Saltillo, bénéficiant d'investissements étrangers, connaissent une croissance démographique dans un contexte de développement industriel important. Queretaro, plus de 900000 habitants, au centre du pays, s'est spécialisée dans la construction mécanique (automobile), l'agroalimentaire et l'électronique, et Hermosillo, plus de 700000 habitants, au nord-ouest, compte une usine automobile Ford et des maquiladoras. Saltillo, 725000 habitants, à l'ouest de Monterrey, surnommée "Little Detroit », avec les usines General Motors et Daimler Chrysler, doit aussi son développement à sa proximité de Monterrey.

En revanche, au sud, les raisons de la croissance peuvent être différentes. Oaxaca, qui dépasse les 500000 habitants, ville de tourisme colonial et préhispanique, de commerces et services, et Tuxtla Gutteriez, ville universitaire importante, sont respectivement les plus grandes villes et capitales de l'Oaxaca et du Chiapas, États les moins urbanisés et les plus pauvres du pays. Leur croissance s'explique moins par l'attractivité économique que par l'accueil d'une émigration rurale encore importante et un croît naturel élevé.

À un troisième niveau de croissance démographique, entre 40 et $60 \%$, toujours en quinze ans, se trouvent huit métropoles intermédiaires, cumulant généralement des fonctions administratives de capitale d’État à une spécialisation économique. Cuernavaca, près de 800000 habitants, capitale du Morelos, à $85 \mathrm{~km}$ de Mexico, a bénéficié de décentralisations dans l'industrie mécanique (usine Nissan), pharmaceutique, électronique, et compte un secteur touristique non négligeable. Aguascalientes, plus de 800000 habitants, près du centre du pays, qui a aussi bénéficié de décentralisations, possède sur son territoire une usine Nissan et un établissement de Texas Instruments.
Chihuahua, près de 800000 habitants, vit des maquiladoras et de l'agroalimentaire. Villahermosa, 650000 habitants, capitale du Tabasco, au sud-est, bénéficie de la pétrochimie. Mérida, capitale de la péninsule du Yucatan, près de 900000 habitants, est une ville de commerces et de services à l'industrie diversifiée et un centre touristique. San Luis Potosi, près d'1 million d'habitants, capitale de l'État du même nom, et Culiacan, 800000 habitants, capitale du Sinaloa, sont des centres commerciaux. Enfin, Mexicali, capitale de la Basse-Californie du Nord, doit son développement notamment aux maquiladoras et à l'agroalimentaire.

\section{Quelques métropoles intermédiaires en moindre croissance}

Quatre métropoles intermédiaires ont une croissance démographique plus faible, inférieure à $40 \%$ en quinze ans, mais néanmoins égale ou supérieure à Mexico. Trois d'entre elles sont maritimes. Sur le golfe du Mexique, Veracruz, avec sa sidérurgie et malgré ses commerces et services et son secteur touristique, ainsi que Tampico, notamment avec sa pétrochimie, sont deux ports qui souffrent de la restructuration de l'industrie lourde. Acapulco, sur la côte Pacifique, connaît la concurrence touristique d'autres stations balnéaires, comme Puerto Vallerta, et d'un arrière-pays rural pauvre, dont la population préfère migrer vers les États-Unis ou le nord du Mexique. Morelia, dans l'État du Michoacan, au centre-est, se situe dans une zone de faible développement et connaît une émigration vers les États-Unis.

Au-dessous des métropoles intermédiaires, les métropoles moyennes, comprenant entre 200000 et 500000 habitants, sont au nombre de 27 selon le dernier recensement de 2005 . Leur nombre s'est accru de dix unités depuis 1990. Leur croissance démographique 1990-2005 (36\%) est inférieure à celle des métropoles intermédiaires, mais néanmoins supérieure à celle de l'agglomération capitale $(22,8 \%)$ et à la moyenne nationale $(27,1 \%)$

\section{Un espace « mexaméricain " ?}

Depuis son entrée dans l'ALENA en 1994, le Mexique apparaît de plus en plus lié économiquement et démographiquement à son grand voisin du Nord : les États-Unis. Du fait de la persistance d'un écart de niveau de vie entre le nord et le sud du Rio Grande, la population des zones rurales, mais aussi des grandes agglomérations, émigre davantage vers les États-Unis, y compris des territoires qui n'avaient guère connu ce processus auparavant, comme l'État de Mexico. Au regard de l'armature urbaine, une rupture est intervenue avec un fort ralentissement de la croissance démographique de la capitale, désormais inférieure à celle des autres agglomérations, comme de la moyenne de la population du Mexique, et donc l'émergence d'une hiérarchie intermédiaire dans l'armature urbaine. À l'échelle régionale, les écarts entre les États à la plus forte croissance démographique et les autres s'accentuent. En conséquence, la géographie du pays semble évoluer, avec un dynamisme renforcé du Nord, participant à la formation d'un espace " mexaméricain », et un ralentissement relatif du Centre, traditionnellement la zone la plus peuplée et la plus dynamique. Le processus de concentration au profit de la mégapole de Mexico semble être contrecarré ${ }^{12}$.

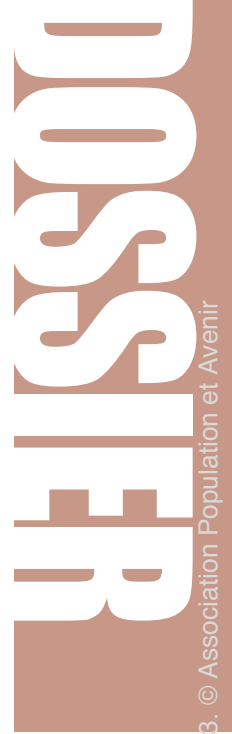

Paris, Éditions Armand Colin, deuxième édition, 2004

7. II y a "macrocéphalie " quand une agglomération d'un pays regroupe $18 \%$ ou plus de a population de ce pays, comme l'aire urbaine de Paris en France, et domine largement 'armature urbaine.

8. Faret, Laurent, Les territoires de la mobilité. Migration et communautés transnationales entre Paris, CNRS Editions, 2003 .

9. Cf. carte des 28 agglomérations mexicaines les plus peuplées page 20 de ce numéro.

10. Usine qui bénéficie d'une exonération des droits de douane pour pouvoir produire dises contes transfordées réparés ou étańs partir de composants importés. partir co composants importes; chandises est ensuite exportée.

1. Par « métropoles intermédiaires ", on peut désigner au Mexique celles qui ne sont pas millionnaires en nombre d'habitants, mais ont un poids démographique supérieur celui des "villes moyennes 》 que l'on situe dans une strate inférieure à 500000 habitants. 12. Cf. également la carte de la 


\section{Le Mexique}

\section{La densité selon les États et l'armature urbaine}

\section{A La densité de la population}

Le peuplement du Mexique (104 millions d'habitants), sur les 1967183 km² du pays, est très diversifié selon les États.

- La densité la plus élevée ( 5817 habitants $/ \mathrm{km}^{2}$ ) est celle du District fédéral dont la superficie est de $1499 \mathrm{~km}^{2}$.

- Les sept États comptant une densité entre 135 et 653 habitants $/ \mathrm{km}^{2}$ sont au centre du Mexique ; la densité la plus élevée est celle de l'État de Mexico (à distinguer du District fédéral), qui compte $21461 \mathrm{~km}^{2}$.

- La densité se situe entre 58 et 112 habitants $/ \mathrm{km}^{2}$ pour sept États dont trois dans le prolongement Ouest du centre du pays, quatre dans le prolongement Est et un au Nord-Est (Nuevo Leon).
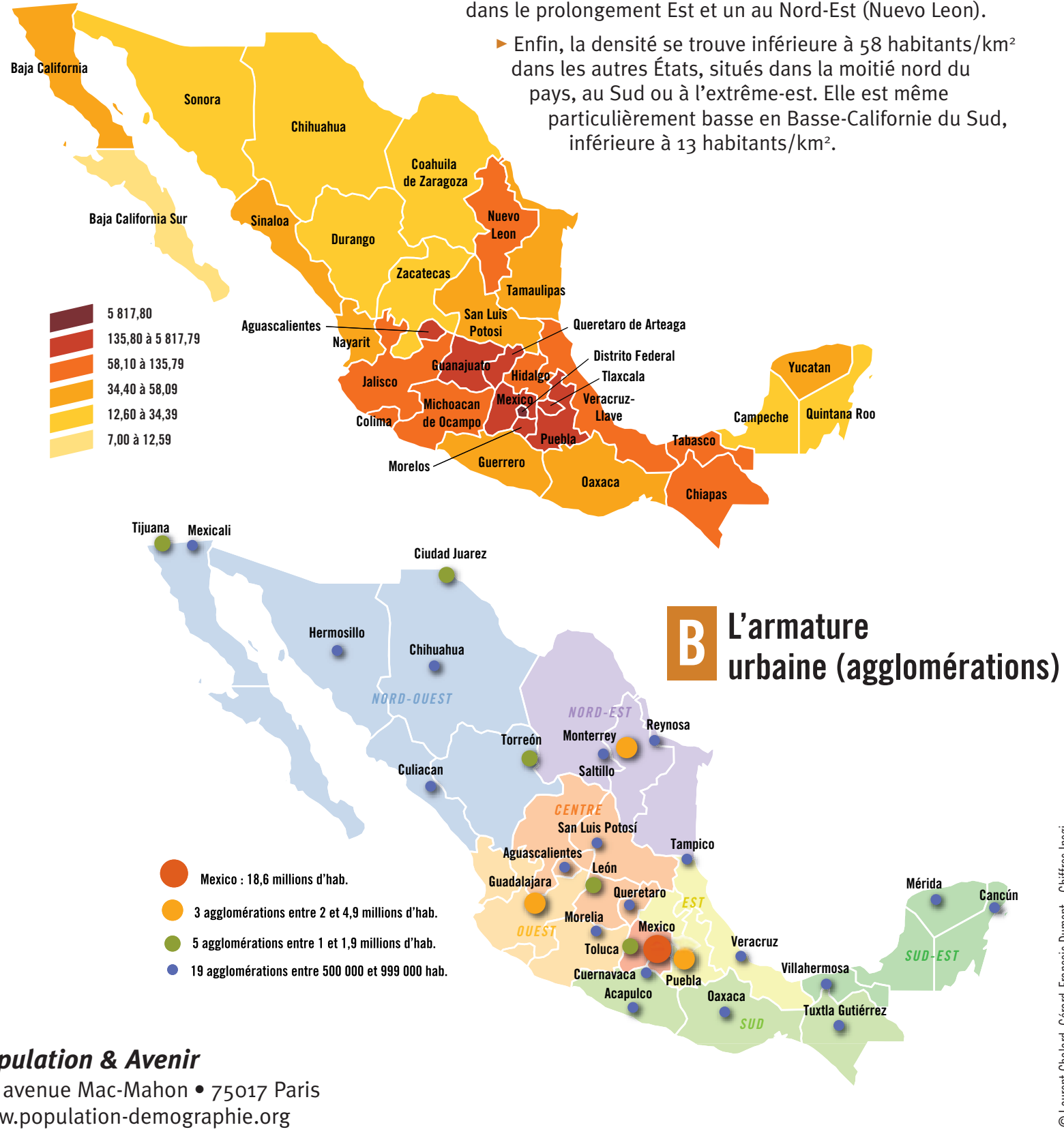

Population \& Avenir

35, avenue Mac-Mahon $\bullet 75017$ Paris www.population-demographie.org 